\title{
Research Paper: Protective Effect of Curcumin on Hippocampal and Behavior Changes in Rats Exposed to Fluoride During Pre- and Post-natal Period
}

\author{
Nagapuri Kiran $\operatorname{Kumar}^{1} \odot$, Mesram Nageshwar ${ }^{1} \odot$, Karnati Pratap $\operatorname{Reddy}^{1^{*}} \odot$
}

1. Department of Zoology, Osmania University, Hyderabad, Telangana, India.

Citation: Kiran Kumar, N., Nageshwar, M., \& Pratap Reddy, K. (2020). Protective Effect of Curcumin on Hippocampal and Behavior Changes in Rats Exposed to Fluoride During Pre- and Post-natal Period. Basic and Clinical Neuroscience, 11(3), 289-300. http://dx.doi.org/10.32598/bcn.11.2.1189.1

http://dx.doi.org/10.32598/bcn.11.2.1189.1

Article info:

Received: 02 Aug 2017

First Revision: 15 Oct 2017

Accepted: 15 Sep 2019

Available Online: 01 May 2020

Keywords:

Curcumin, Sodium

fluoride, Neurotransmitters, Hippocampus, Behavior

\section{A B S T RA C T}

Introduction: Curcumin, a yellow-pigment, found in the popular Indian spice turmeric (Curcuma longa), poses pharmaceutical applications due to its anti-inflammatory, antioxidant, and chemoprotective properties. Excessive fluoride causes fluorosis leading to neurodegeneration and associated behavioral deficits, particularly in children. This study aimed at investigating the neuroprotective ability of curcumin on sodium fluoride (NaF)related alterations of acetylcholine, catecholamines, histological changes in hippocampus and behavior of rats exposed to $\mathrm{NaF}$ during pre- and post-natal period.

Methods: Pregnant albino Wistar rats were chosen and divided into four groups. The experimental period lasted 53 days (i.e. the gestational period of 23 days and post-gestational period of 30 days), at which the control group received normal tap water, the experimental group received $\mathrm{NaF}(20 \mathrm{ppm} / \mathrm{kg}$ bw) through drinking water, and the protective groups received curcumin $(10 \mathrm{mg}$ and $20 \mathrm{mg} / \mathrm{kg}$ bw $)$ by gavage and $\mathrm{NaF}(20 \mathrm{ppm} / \mathrm{kg}$ bw) through drinking water. Behavioral study (open field test) was done using postnatal pups aged 21 and 30 days. The brains of postnatal pups aged 1, 7, 14, 21, and 30 days were collected and used for biochemical analysis and those of pups aged 14, 21, and 30 days were used for histopathological analysis.

Results: NaF-exposed rats showed a significant $(\mathrm{P}<0.05)$ decrease in body weight, brain weight, and behavioral activities, which were significantly reversed with curcumin treatment. The levels of epinephrine significantly $(\mathrm{P}<0.05)$ increased, whereas norepinephrine, dopamine and acetylcholine levels declined in NaF-treated group compared with the control group, which were significantly $(\mathrm{P}<0.05)$ reversed after treatment by curcumin $(10 \mathrm{mg} / \mathrm{kg}$ bw and 20 $\mathrm{mg} / \mathrm{kg}$ bw) along with $\mathrm{NaF}$. The histological alterations, including shrinkage of neurons and nissal substances were observed in the hippocampus of $\mathrm{NaF}$-treated pups that the control pups, whereas co-treatment with curcumin and $\mathrm{NaF}$ showed ameliorative effects and controlled the histological alterations.

Conclusion: The results showed the neuroprotective effect of curcumin on behavior, neurotransmitter levels, and histological changes in the hippocampus against $\mathrm{NaF}$-induced neurotoxicity in developing rat pups. 


\section{Highlights}

- Curcumin shows neuroprotection against NaF-induced toxicity during development of brain particularly hippocampus.

- Curcumin reversed developmental exposure of NaF-induced decrease in body weight, brain weight and behavioural responses in rats.

- The NaF-induced altered levels of neurotransmitters epinephrine, norepinephrine, dopamine and acetylcholine were reversed with curcumin during development of brain of rat.

- The developmental exposure of NaF-induced histological alterations in hippocampus were ameliorated with cotreatment of curcumin.

- The dosage of curcumin at $20 \mathrm{mg} / \mathrm{kg}$ bw showed better mitigating ability than that of $10 \mathrm{mg} / \mathrm{kg}$ bw.

\section{Plain Language Summary}

Fluorosis, results with excessive intake of fluoride, is a progressive degenerative disorder in human beings affecting skeletal and non-skeletal systems particularly with known neurotoxic effects. Excessive intake of fluoride through water, food, toothpaste, pollutants and other sources is a serious health problem. This is more prevalent in India and china which are endemic and other parts of world where fluoride levels exists between 1-29 ppm. Studies also revealed that neurotoxin show severe degenerative effects on growing children, where mother and child were continuously exposed, resulting in cognitive, behavioural and motor deficits in children as well as grown up adults. On the other hand, there is no successful solutions are available to prevent fluorosis, except suggestive intake of calcium, magnesium, vitamin $\mathrm{C}, \mathrm{D}$, selenium and certain natural compounds with antioxidant potential. The present study evaluated the protective effects of co-treatment of curcumin against developing brain exposed to $\mathrm{NaF}$ during pre and post natal period with reference to brain, behaviour and histological as well as neurotransmitter system of hippocampus of rat, a center related to learning and memory. Curcumin is an active flavonoid, used as a food additive, important component of India and Chinese medicine with antioxidant, antiinflammatory, antidiabetic etc properties. The results suggest that cotreatment of curcumin with developmental exposure of fluoride in rats show reversal of fluoride induced behavioural, neurotransmitter's alterations and histological changes of hippocampus of brain. Thus, it is established from this study that curcumin exhibits neuroprotective effects against development of fluorosis, particularly in relation to brain, behaviour and cognition. The dosage of $20 \mathrm{mg} / \mathrm{kg}$ bw of curcumin is more effective then $10 \mathrm{mg} / \mathrm{kg}$ bw of curcumin.

\section{Introduction}

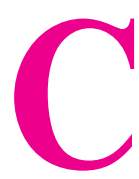

urcumin is an active flavonoid in the Curcuma longa Linn, found in the rhizome of the plant extensively used as a food additive and a constituent in drugs and cosmetics with a broad range of effects. Curcumin is an important component of Indian and Chinese medicine (Hemlata \& Mandava, 2010). Previous reports on the use of natural and complementary medicine in addition to modern medicine have drawn the attention of the scientific community towards natural products. Curcumin has an amazingly wide range of compounds with antioxidant, antimicrobial, antihepatotoxic, hypolipidaemic, anti-inflammatory, and anti-diabetic proper- ties (Ying et al., 2005; Nabavi, Eslami, Hajizadeh, \& Nabavi, 2011; Joyce et al., 2013). Its antioxidant properties through oxygen free radicals and inhibition of lipid peroxidation have been reported in animal studies (Majid et al., 2015; Nabavi, Moghaddam, Eslami, \& Nabavi, 2012; Nabavi, Moghaddam, Nabavi, \& Eslami, 2011).

Curcumin is a more potent antioxidant than any other antioxidant nutrients, such as $\beta$-carotene and vitamins $\mathrm{E}$ and C (Suresh \& Srinivasan, 2007). Chronic administration of curcumin has been reported to exert a neuroprotective-like effect in rat brain (Shrinivas, Mohit, \& Mahendra, 2008). Curcumin has found with antidepressant function by assisting the role of monoaminergic systems and controlling the release of neurotransmitters, such as 5-Hydroxyindoleacetic Acid (5-HIAA), epinephrine, norepinephrine, and dopamine 
(Xiaolie et al., 2016). It also inhibits inflammatory cytokine production, platelet aggregation, cyclooxygenase, lipoxygenase isoenzymes, protein kinase- $\mathrm{C}$, protein tyrosine kinases, calcium-dependent endonuclease, and apoptosis (Mahendra, Kanwaljit, \& Shrinivas, 2008).

Fluorosis is a progressive degenerative disorder in humans and animals. Fluoride is an essential trace element but its excessive intake can cause damage to both skeletal and nonskeletal systems. Excessive intake of sodium fluoride $(\mathrm{NaF})$ through food, water, toothpaste, pollutants, and other sources is a serious health problem, particularly during child growth. In this situation, the Blood-Brain Barrier (BBB) fails to regulate the fluoride entry into the brain making it more susceptible to metabolic alterations at the subcellular level (Madhusudhan, Basha, Rai, Ahmed, \& Prasad, 2010).

The high fluoride ingestion leads to neuronal degeneration resulting in Central Nervous System (CNS) dysfunction (Geeraerts, Gijs, Finne, \& Crokaert, 1986). Earlier reports on the effects of $\mathrm{NaF}$ on rat brain and neuronal cell bodies in the hippocampus suggest that excess fluoride intake affects CNS (Vani \& Reddy, 2000; Bhatnagar et al., 2006; Chirumari \& Reddy, 2007; Yugandhar, Reddy, \& Praveen, 2011). Free radical scavenging enzyme activities are lower in people living in the endemic fluorosis areas making them more prone to oxidative damage (Li \& Cao 1994; Shivarajashankara, Shivashankara, Gopalakrishna, \& Rao, 2001).

Fluoride can interfere with brain function, reducing Intelligence Quotient (IQ) levels in children, cognition, memory, and locomotor behavior (Li \& Gao, 1995). Chronic fluoride administrated in pregnant mothers can lead to deleterious effects in their offspring, as fluoride can pass across the placenta and BBB of the fetus hindering the brain development, altering brain tissue morphology, neuron ultrastructure, and neurotransmitters content (Madhusudhan et al., 2010). In our previous studies, the potential benefits of natural compounds, such as Resveratrol (Chandrashakar \& Reddy, 2016), Quercetin (Nageshwar, Kirankumar, Rajkiran, Chandrashakar, \& Reddy, 2017), and the extract of Abelmoschus moschatus (Sudhakar, Nageshwar, \& Reddy, 2017) against NaF-induced neurotoxicity in rats' brain were observed. Recently, we have also reported that curcumin reversed the free radicals generation in rats exposed to $\mathrm{NaF}$ (Nageshwar et al., 2017). The developmental fluoride exposure crosses the BBB due to the immaturity of BBB (Varner, Jensen, Horvath, \& Issacon, 1998). The continued exposure of fluoride alters the integration of blood vascular system in brain leading to deprivation of the brain nourishment and oxygen supply to brain (Mullenix, Denbesten, Schunior, \& Kernan, 1995).
Lu et al. (2000) reported children living in endemic fluorosis areas with a significantly lower IQ compared with nonendemic regions. Fluoride is considered a health problem in endemic areas, as well as in other populations exposed to fluoride; however, no successful solutions are available to prevent fluorosis, particularly the postnatal period. Considering the potential of using new compounds in the treatment of fluorosis, this study aimed at reporting the protective effect of curcumin in NaF-induced behavioral and histological changes, as well as changes in catecholamines and acetylcholine $(\mathrm{ACh})$ in the developing hippocampus of rats.,

\section{Methods}

\subsection{Chemicals}

Curcumin, epinephrine, norepinephrine, and dopamine were purchased from the Sigma-Aldrich chemicals. NaF, acid butanol, heptane, sodium acetate, perchloric acid, $\mathrm{FeCl} 3$, HCL, EDTA, glacial acetic acid, cresyl violet stain, and other chemicals were purchased from local chemical suppliers (Himedia).

\subsection{Experimental procedure}

Twenty-four timed-pregnant albino rats (Wister) were received from NIN, Hyderabad, India. The pregnant rats were maintained under standard laboratory conditions in single rat polypropylene cages. The standard diet and drinking water were provided ad libitum. The ethical procedures were performed following the guidelines of CPCSEA at Osmania University, Hyderabad, India. Timed-pregnant rats aged 120150 days were randomly divided into four groups of 6 rats.

The first group was served as the control group and received pure drinking water. The second group was served as the experimental group and received drinking water containing 20 ppm of $\mathrm{NaF}$. The third and fourth groups also were considered as protective groups and received curcumin 10 and 20 $\mathrm{mg} / \mathrm{kg}$ bw, respectively, through oral gavage along with 20 ppm $\mathrm{NaF}$ by drinking water. The rats were treated for 53 days (i.e. 23 days during gestation and 30 days postpartum).

One-day pregnant rats were allowed to take $20 \mathrm{ppm} \mathrm{NaF}$ and curcumin (prenatal exposure or gestational exposure) up to the end of the experimental period (53 days), one-day weanling rat pups ( $22^{\text {nd }}$ day of the experiment) and 21-day weanling rat pups ( $43^{\text {rd }}$ day of the experiment) received $\mathrm{NaF}$ and curcumin through lactation by mother, and 22-day adult rat pups ( $44^{\text {th }}$ day of the experiment) 30 -day adult rat pups (53 day of the experiment) received $\mathrm{NaF}$ by drinking water and curcumin by oral gavage. Postnatal pups of 1, 7, 14, 21 and 30 days of age were selected for experimental studies. 
The brains were dissected and stored at $20^{\circ} \mathrm{C}$. The biochemical assays were conducted on brains and $10 \%$ formaldehyde used to store the brains for histopathological examination. The behavioral studies were conducted on 21- and 30-day rat pups.

\subsection{Body weight and brain weight}

The postnatal pups' brain weight and body weight at different ages in different groups were recorded.

\subsection{Behavioral study: Open Field Test (OFT)}

The OFT was used to measure behavioral responses, such as locomotor activities, anxiety, hyperactivity, and exploratory behaviors using the method developed by Netto, Dias, \& Izquiedo, (1986). The apparatus consisted of 36 identical squares of $20 \mathrm{~cm}$ per side $(90 \mathrm{~cm} \times 90 \mathrm{~cm} \times 30 \mathrm{~cm})$ made of wood. Each animal was placed in the corner of the field and allowed to explore the field for $5 \mathrm{~min}$. The behavioral activities, such as crossing, rearing, rearing on the wall, sniffing, and grooming were counted as the sum of all open-field tasks (total behavior), and the obtained results were reported as Number of entries (counts/5 min).

\subsection{Biochemical assays: Neurotransmitters}

\subsection{1. $\mathrm{ACh}$}

ACh level was estimated based on the Hestrin Method (1949). Accordingly, $10 \mathrm{mg}$ of the hippocampus was weighed and homogenized by adding $1 \mathrm{~mL}$ of $3 \%$ perchloric acid and centrifuged at $1200 \mathrm{rpm}$ for $10 \mathrm{~min}$. The supernatant was used for the estimation. Hydroxylamine reagent was prepared freshly by mixing the equal volumes of reagents 1 and 2 and incubated for $3 \mathrm{~h}$ at room temperature. Next, $2 \mathrm{ml}$ of alkaline hydroxylamine reagent was added to $1.0 \mathrm{ml}$ of the supernatant, and before adding $1 \mathrm{ml}$ of $\mathrm{FeCl} 3$ solution, the $\mathrm{pH}$ was adjusted to 1.5 by adding $0.001 \mathrm{~N} \mathrm{HCL}$ and mixed well by vortexing. The optical density was measured at 540 $\mathrm{nm}$. The results were expressed as $\mathrm{mg}$ of $\mathrm{ACh} / \mathrm{gm}$ wet weight tissue.

\subsection{Preparation of the monoamine sample}

The brain tissue was homogenated in $0.25 \mathrm{M}$ sucrose solution and centrifuged at $4^{\circ} \mathrm{C}$ at $1000 \mathrm{rpm}$ for $10 \mathrm{~min}$. The samples were prepared by the procedure described previously (Nageshwar et al., 2017). The precipitate (acid alumina) was used for the catecholamines assay (Kari, Davidson, Kohl, \& Kochhar, 1978).
2.7. Catecholamines: Epinephrine, norepinephrine, and dopamine

A modified protocol (Kari el al., 1978) was used to estimate the levels of Epinephrine, Norepinephrine, and Dopamine using a fluorometer (Jasco FP750). The alumina was washed by vortexing the tube twice with $2 \mathrm{ml}$ of double-distilled water and centrifuged at $1000 \mathrm{~g}$ for $5 \mathrm{~min}$. The water was removed and the alumina was shaken for $5 \mathrm{~min}$ with $2 \mathrm{ml}$ of acetic acid $0.2 \mathrm{~N}$ for elution of the catecholamines.

The tubes were centrifuged at $1000 \mathrm{~g}$ for $10 \mathrm{~min}$ and $1 \mathrm{ml}$ of the supernatant liquid was transferred to another tube. Then, $0.1 \mathrm{ml}$ of EDTA $0.1 \mathrm{M}$ reagent was added to adjust the $\mathrm{pH}$ to 6.3 , and $0.1 \mathrm{ml}$ of $0.1 \mathrm{~N}$ iodine solution was added and mixed thoroughly, and allowed to stand for $2 \mathrm{~min}$. At the end of $2 \mathrm{~min}$, alkaline sodium sulphite solution was added and the mixture allowed standing for $2 \mathrm{~min}$ at room temperature. The readings were noted for Epinephrine with an excitation at $410 \mathrm{~nm}$ and emission at $500 \mathrm{~nm}$. Norepinephrine level was estimated after heating the same solution for $2 \mathrm{~min}$ in a boiling water bath. The tubes were cooled and readings were noted for norepinephrine with an excitation at $387 \mathrm{~nm}$ and Emission at $487 \mathrm{~nm}$.

The solution was then used for dopamine analysis. The solution was heated for $5 \mathrm{~min}$ in a boiling water bath. After cooling the tubes, the fluorescence of dopamine was read using a fluorometer with an excitation of $310 \mathrm{~nm}$ and emission of $410 \mathrm{~nm}$. The values were expressed as $\mu \mathrm{g}$ amine/gm wet weight of tissue.

\subsection{Histopathology}

The brain sections were fixed in freshly prepared $10 \%$ formalin, processed routinely, and embedded in paraffin. The 5 $\mu \mathrm{m}$-thick sections were made and stained with cresyl violet. This staining method determines the neural cell density and is used for histopathological examination (Pilati, Matthew, Sofoklis, Revers, \& Martine, 2008). The tissue sections were transferred onto the $0.5 \%$ gelatin-coated slides without wrinkles. The slides were stored overnight in a dust-free chamber. The next day, they were deparaffinized with xylene I and II for $30 \mathrm{~min}$, then hydrated with DW in the following sequences: $100 \%, 90 \%, 70 \%, 50 \%, 30 \%$ for $10 \mathrm{~min}$. The slides were kept into a cresyl violet stain solution for 5-15 min followed by washing with running tap water for $10 \mathrm{~min}$. Dehydration of the sections was performed with $30 \%, 50 \%$, $70 \%, 90 \%$ alcohol for $10 \mathrm{~min}$. The slides were subsequently transferred into $1 \%$ eosin for $30 \mathrm{~s}$. Then, they were kept in $100 \%$ alcohol and cleared with Xylene I and II for $10 \mathrm{~min}$, air-dried, mounted in DPX, and examined using an Olympus microscope (Magnification $40 \mathrm{X}$ ). 


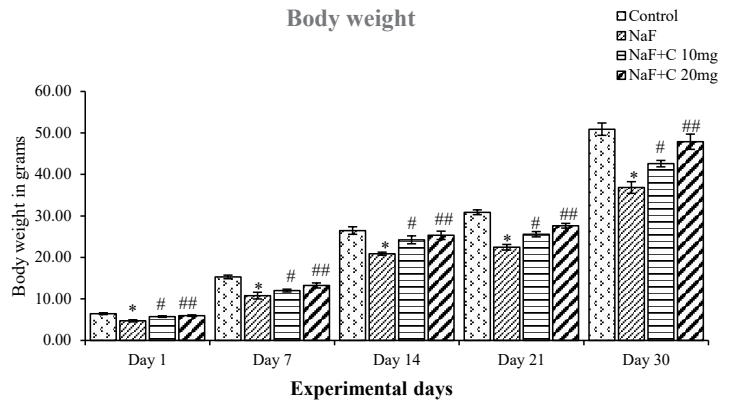

NEUR SSCIENCE

Figure 1. Effect of curcumin on body weight of the rats treated with sodium fluoride $(\mathrm{NaF})$.

* $\mathrm{P}<0.05$ vs. the control group. ${ }^{\mathrm{P}}<0.05$ and ${ }^{\# \# P}<0.05$ vs. the $\mathrm{NaF}$-treated group. Values are expressed as Mean $\pm \mathrm{SD}$ and the results are reported in grams $(\mathrm{g}) ;(\mathrm{n}=6)$.

\subsection{Statistical analysis}

The values are expressed as Mean $\pm \mathrm{SD}$ and Mean \pm SEM. A comparison of the means was conducted using the two-way analysis of variance. The significant level was considered at $\mathrm{P}<0.05$. The SPSS V. 20 software was applied for statistical analyses.

\section{Results}

The body and brain weight of rats on $1,7,14,21$, and 30 days of treating with $\mathrm{NaF}$ significantly $(\mathrm{P}<0.05)$ decreased compared with the control group and the groups treated with curcumin and $\mathrm{NaF}$ showed a reversal in the body and brain weight compared with the $\mathrm{NaF}$ group (Figures $1 \& 2$ ). The rats treated with curcumin $(20 \mathrm{mg} / \mathrm{kg} \mathrm{bw})$ showed a better mitigating ability $(\mathrm{P}<0.05)$ than those treated with curcumin $(10 \mathrm{mg} / \mathrm{kg}$ bw) against $\mathrm{NaF}$ toxicity; however, both curcum-

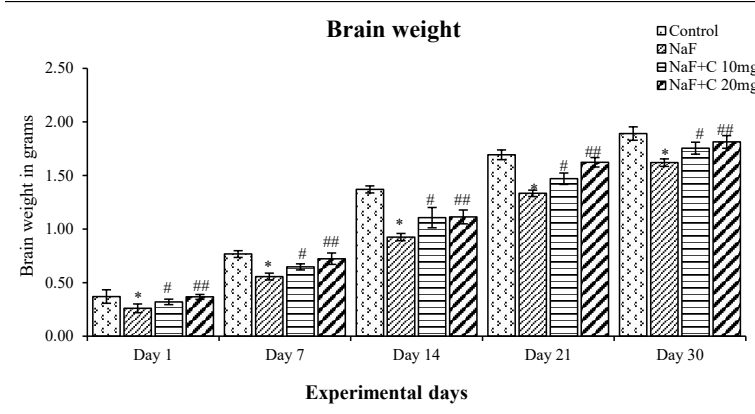

NEUR $\$$ SCIENCE

Figure 2. Effect of curcumin on brain weight of the rats treated with sodium fluoride $(\mathrm{NaF})$.

${ }^{*} \mathrm{P}<0.05$ vs. the control group. ${ }^{\#} \mathrm{P}<0.05$ and ${ }^{\# \#} \mathrm{P}<0.05$ vs. NaFtreated group. Values are expressed as Mean \pm SD and the results are reported in grams $(\mathrm{g}) ;(\mathrm{n}=6)$.

in concentrations showed a positive and protective effect on $\mathrm{NaF}$ toxicity.

The locomotor activity significantly $(\mathrm{P}<0.05)$ decreased in the NaF-treated group than the control group, whereas it significantly $(\mathrm{P}<0.05)$ increased in the curcumin-treated groups in comparison with the $\mathrm{NaF}$ group (Figure 3). Both 10 and $20 \mathrm{mg} / \mathrm{kg}$ bw curcumin doses were effective against $\mathrm{NaF}$ toxicity. Regarding locomotor activity, the rats treated with curcumin $(20 \mathrm{mg} / \mathrm{kg}$ bw)showed a better mitigating capability $(\mathrm{P}<0.05)$ than those treated with curcumin $(10 \mathrm{mg} / \mathrm{kg} \mathrm{bw})$ against $\mathrm{NaF}$ toxicity.

The ACh levels significantly $(\mathrm{P}<0.05)$ reduced in the NaFtreated group in post-natal pups aged $1,7,14,21$, and 30 days compared with the control group, and the acetylcholine levels were significantly $(\mathrm{P}<0.05)$ reversed as that of the control group in curcumin-received groups than the NaF-

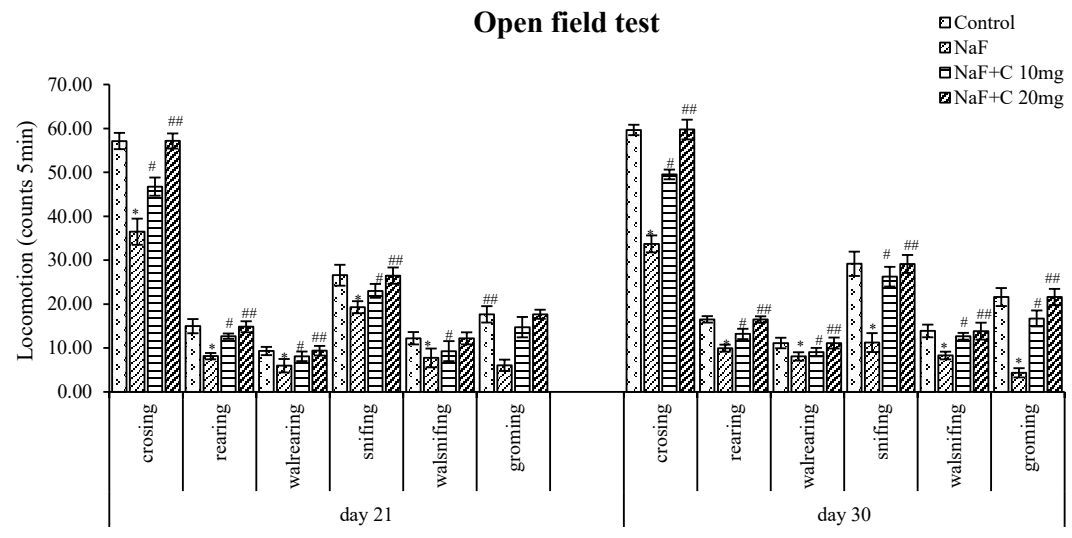

Figure 3. Effect of curcumin on Open field test results in rats treated with sodium fluoride.

${ }^{*} \mathrm{P}<0.05$ vs. the control group. ${ }^{\#} \mathrm{P}<0.05$ and ${ }^{\# \#} \mathrm{P}<0.05$ vs. sodium fluoride $(\mathrm{NaF})$-treated group. Values are expressed as Mean \pm SEM and the results are reported as the number of entries (counts $/ 5 \mathrm{~min}) ;(\mathrm{n}=6)$ 


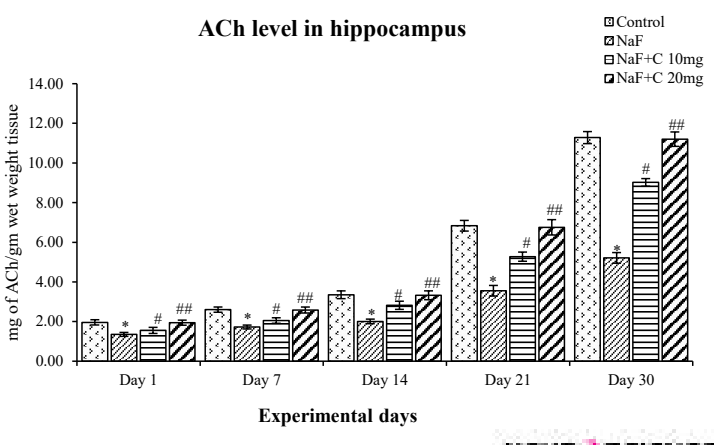

NEUR JSCIENCE

Figure 4. Acetylcholine levels in the developing hippocampus of the rats treated with sodium fluoride $(\mathrm{NaF})$ and curcumin.

${ }^{*} \mathrm{P}<0.05$ vs. the control group; ${ }^{\#} \mathrm{P}<0.05$ and ${ }^{\# \# ~} \mathrm{P}<0.05$ vs. NaFtreated group. Values are expressed as Mean \pm SEM and the results are reported in $\mathrm{mg}$ of $\mathrm{ACh} / \mathrm{gm}$ wet weight tissue; $(\mathrm{n}=6)$.

treated group (Figure 4). The epinephrine levels significantly $(\mathrm{P}<0.05)$ increased an age-dependent manner in 1-day-old rat pups than to 30-day-old rat pups treated with $\mathrm{NaF}$ compared with the control group, and the epinephrine levels were significantly $(\mathrm{P}<0.05)$ restored in the curcumin-received groups than the NaF-treated group (Figure 5). The norepinephrine and dopamine levels significantly $(\mathrm{P}<0.05)$ decreased in rat pups aged 1, 7, 14, 21, and 30 days treated with $\mathrm{NaF}$ than the controls. However, the norepinephrine and dopamine levels were significantly $(\mathrm{P}<0.05)$ restored in the curcuminreceived groups compared with the $\mathrm{NaF}$ group (Figs. 6 and 7). In this regard, the curcumin-administered rats $(20 \mathrm{mg} / \mathrm{kg}$ bw) showed a better mitigating ability $(\mathrm{P}<0.05)$ than the curcumin-administered rats $(10 \mathrm{mg} / \mathrm{kg} \mathrm{bw})$ against $\mathrm{NaF}$ toxicity.

The histopathological alterations were observed in the CA1 and $\mathrm{CA} 2$ regions in the hippocampus of the postnatal rat pups (aged 14, 21, and 30 days) using cresyl violet stain. The control group was found with neurons with normal morphology.

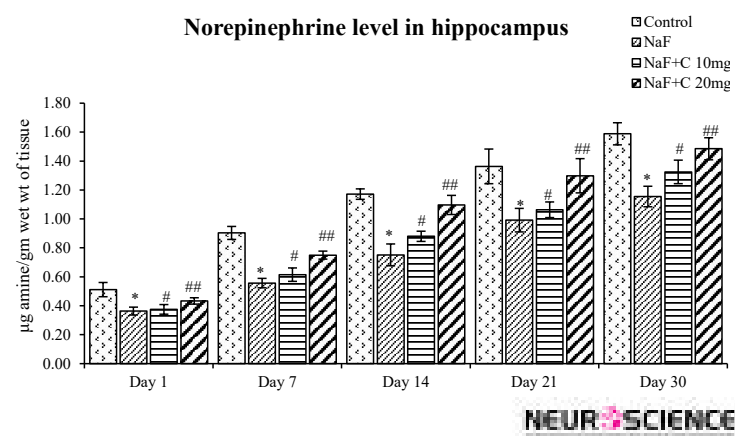

Figure 6. Norepinephrine levels in the developing hippocampus of rats treated with sodium fluoride $(\mathrm{NaF})$ and curcumin

${ }^{*} \mathrm{P}<0.05$ vs. the control group; ${ }^{\#} \mathrm{P}<0.05$ and ${ }^{\# \#} \mathrm{P}<0.05$ vs. the NaFtreated group. Values are expressed as Mean $\pm \mathrm{SEM}$ and the results are reported in $\mu \mathrm{g}$ amine/gm wet wt of tissue; $(n=6)$.

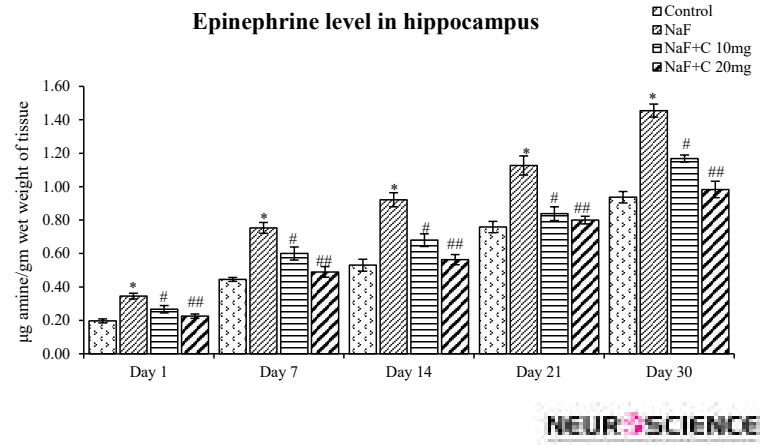

Figure 5. Epinephrine levels in the developing hippocampus of the rats treated with sodium fluoride $(\mathrm{NaF})$ and curcumin

${ }^{*} \mathrm{P}<0.05$ vs. the control group. ${ }^{\#} \mathrm{P}<0.05$ and ${ }^{\# \#} \mathrm{P}<0.05$ vs. the $\mathrm{NaF}$-treated group. Values are expressed as Mean \pm SEM and the results are reported in $\mu \mathrm{g}$ amine/gm wet weight of tissue; $(n=6)$.

The neural cells' density, population, and arrangement gradually decreased and disturbed in the NaF-treated group in comparison with the control group. Curcumin-administered groups showed healthy neurons and good cell arrangement and density compared with the NaF-treated group (Figure 8). The curcumin at a dose of $20 \mathrm{mg} / \mathrm{kg}$ bw was more effective than curcumin at a dose of $10 \mathrm{mg} / \mathrm{kg}$ bw against $\mathrm{NaF}$-induced histological alterations.

\section{Discussion}

The results of this study showed that $\mathrm{NaF}$ intake induced significant changes in neurotransmitters and caused behavioral and histological alterations in the hippocampus of the developing brain of rats. The recovery of body weight and brain weight of NaF-treated rats with curcumin agrees with earlier studies (Nageshwar et al., 2017). The decreased body and brain weight might be due to reduced food intake or dis-

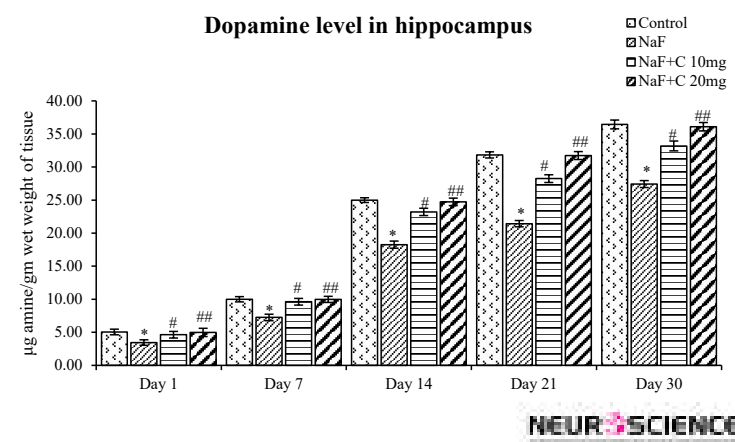

Figure 7. Dopamine levels in developing hippocampus of the rats treated with sodium fluoride $(\mathrm{NaF})$ and curcumin.

* $\mathrm{P}<0.05$ vs. the control group; ${ }^{*} \mathrm{P}<0.05$ and ${ }^{\# \# ~} \mathrm{P}<0.05$ vs. the NaF-treated group. Values are expressed as Mean \pm SEM and the results are reported in $\mu \mathrm{g}$ amine/gm wet weight of tissue; $(n=6)$. 


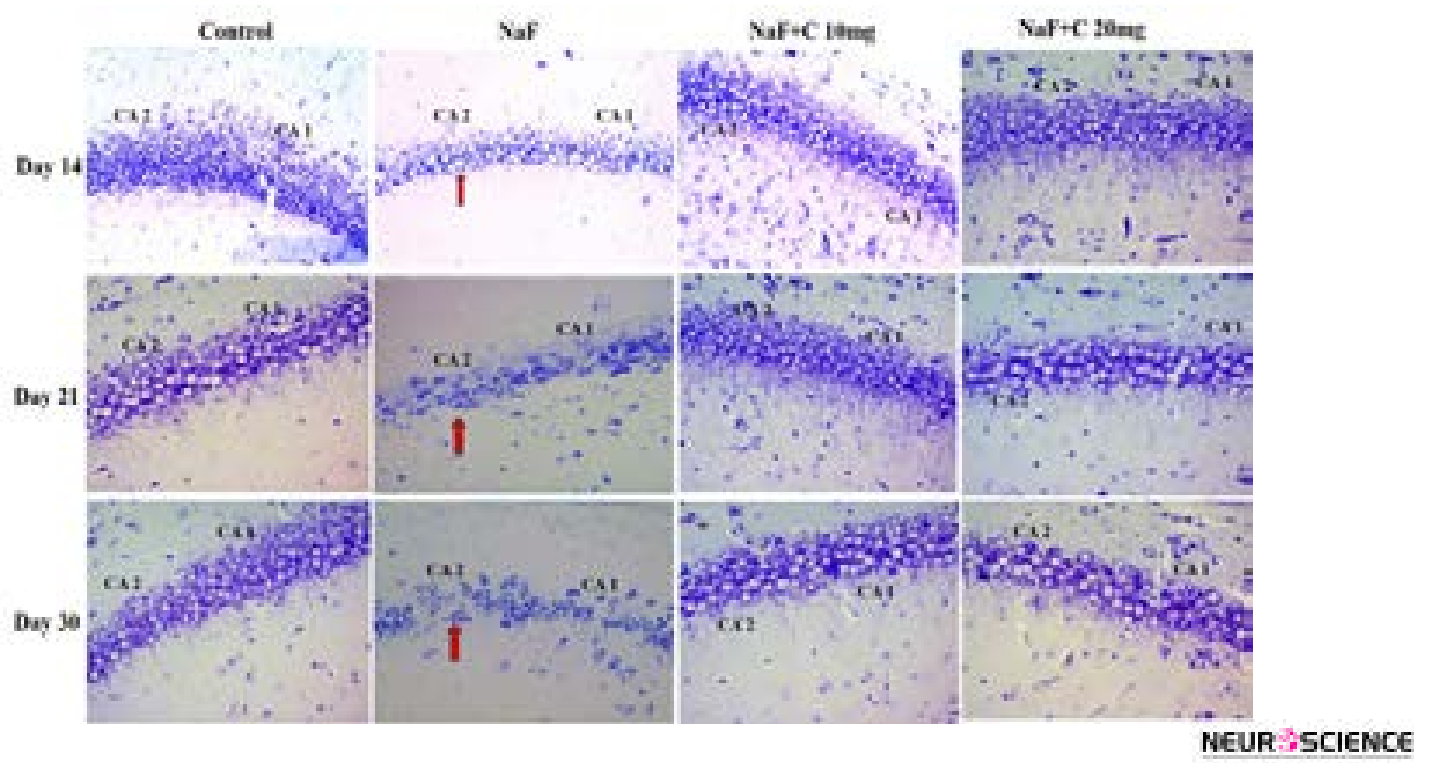

Figure 8. Histopathological studies in the hippocampal CA1 and CA2 regions stained by cresyl violet in the control group and groups treated with sodium fluoride $(\mathrm{NaF}), \mathrm{NaF}+$ curcumin $(10 \mathrm{mg})$, and $\mathrm{NaF}+$ curcumin $(20 \mathrm{mg})$ on postnatal days 14,21 , and 30. The neurons were arranged closely, had a circular shape, and the nuclear membrane was clear in the hippocampal CA1 and $\mathrm{CA} 2$ regions of the control group and groups treated with $\mathrm{NaF}+$ curcumin $(10 \mathrm{mg})$ and $\mathrm{NaF}+$ curcumin $(20 \mathrm{mg})$ groups. Arrow marks show the neurons with irregular and shrunken shapes, and the nuclear membrane was indistinct in the CA1 and CA2 regions in the NaF-treated group (Magnification 40X).

turbed protein and energy metabolism after fluoride ingestion (Chinoy, Sequeria, \& Narayana, 1991).

Fluoride alters the activities of the enzymes, free radical generation, lipid peroxidation, and protein oxidization factors, which may also influence the decrease in brain weight due to fluoride exposure (Vani \& Reddy, 2000; Shantakumari, Srinivasulu, \& Subramanian, 2004; Rajkiran \& Reddy, 2015). Curcumin has a reducing effect on toxicant-induced physiological changes in animals (Shamsher, Sumit, \& Puneet, 2015). Curcumin has some protective roles against fluoride because of its antioxidant capacity to minimize the breakdown of proteins and cell disturbances, evidenced by normal body weight and brain weight observed in the curcumin-administered groups.

The neurotransmitters levels altered in the hippocampus and neocortex region of the brain of adult rats exposed to fluoride (Chirumari \& Reddy, 2007; Wu, Gu, Ge, Zhang, \& Wang, 2006). Exposure to fluoride also results in a decrease in AChE in the brain of the rats and mice (Wang, Ge, Ning, \& Wang, 2004). Shan et al. (2004) reported a decrease in nicotinic acetylcholine receptors and injury to the post-transcription factor of the receptor gene by oxidative stress. Nacetylcholine receptors play an important role in learning and memory (Brown, Buckingham, \& Jones, 2009). The level of Ach decreased in the developing brain of rats treated with $\mathrm{NaF}$ (Nageshwar et al., 2017). Rajkiran et al. (2018) also reported changes in Ach levels in fluoride-intoxicated rats.
The cholinesterase inhibition with fluoride exerts a toxic effect in the brain regions due to abruption in the cholinergic transmission leading to decreased learning and memory $(\mathrm{Lu}$, Sun, Wu, Wang, Lu, \& Liu, 2000). Chronic exposure to NaF reduces acetylcholinesterase (AChE) activity in the rat brain (Ekambaram \& Vanaja, 2001). Curcumin with antioxidative effects restores depleted neurotransmitters levels in 3-nitropropionic acid-exposed rats (Shamsher, Sumit, \& Puneet, 2015). The observed reversal of depleted acetylcholine level with curcumin following fluoride exposure in developing rats has reported earlier.

Monoaminergic system plays an important role in the establishment of neuronal circuits during the development of brain. Catecholamines in addition to their effect as neurotransmitters can influence metabolism (Ying et al., 2005). A substantial increase in dopamine and a disturbance in catecholamine metabolism following fluoride administration have observed in the hippocampus (Chirumari \& Reddy, 2007). The changes in biogenic amine levels due to fluoride exposure affect the rate of turnover of biogenic amine enzymes, which in turn has a glutaminergic effect on other neurotransmitters. (Nageshwar et al., 2017) reported a decrease in epinephrine levels after treatment with flavonoids and an increase in the developing brain of the rats exposed to $\mathrm{NaF}$. $\mathrm{Xu}$ et al. (2005) observed an improvement in the levels of catecholamines in the brain of animals treated with curcumin. Also, norepinephrine levels decreased after treatment with $\mathrm{NaF}$ (Nageshwar et al., 2017) and increased after treatment 
with curcumin (Mohit, Mahendra, \& Shrinivas, 2009), which is consistent with our observations. Marcela, Patricia, Estela, Lea, Claudio \& Roberto, (2011) also observed that chronic $\mathrm{NaF}$ intake altered the levels of neurotransmitters, such as norepinephrine and serotonin (5-HT) in rat brain. Our results on the developing brain can be more determined through an earlier observation on the adult brain and indicated the neuroprotective ability of curcumin modulation on central monoaminergic neurotransmitter systems.

The activities of locus coeruleus of the brainstem are mediated through norepinephrine, which monitors external stimuli and responses, such as fight-or-flight and pain. The noradrenergic inputs to the neocortex, hippocampus, thalamus, septum, cerebellum, and brainstem are provided by locus coeruleus. Norepinephrine is important for attention, arousal, and learning, any changes in its levels lead to Parkinson disease, Alzheimer disease, attention deficit disorder, hyperactivity, post-traumatic stress disorder, and disturbed hypothalamic function (Brown et al., 2009).

The cognition, mood, emotions, movement and blood pressure are controlled through norepinephrine and locus coeruleus. The vast majority of dopaminergic cells arise from the midbrain; however, some also arise from the diencephalon. The dopaminergic lesions cause cognitive dysfunction, poor working memory skills (Itmori et al., 1982), attention deficit, and altered locomotor activity (Seibenhener \& Wooten, 2015). Ying et al. (2005) also reported the role of dopamine in schizophrenia, Parkinson disease, Huntington chorea, manic-depressive illness, and tardive dyskinesia. Alterations in catecholamines, indoleamine, and their metabolites cause toxicity in the CNS (Tsunoda, Wakano, Horiuchi, Itai, \& Tsunoda, 2005). Mohit et al., (2009) demonstrated that the dopamine levels decreased in toxicant-injected animal models. Curcumin dose-dependently ameliorated the neurotransmitters and molecular alterations against reserpine-induced pain/depression in animals (Arora, Kuhad, \& Tiwari, 2011). Shamsher et al., (2015) also found the improved level of dopamine in the brain of rats treated with curcumin, and this effect was attributed to neurotransmitters modulating the activity of curcumin.

Fluoride inhibits spontaneous motor activity, which includes locomotor, as well as scratching and grooming activities through the impairment of cholinergic mechanism (Wang et al., 2004; Ying et al., 2005; Ekambaram \& Vanaja, 2001). The deficit in motivation may lead to the suppression of the eating process and a decreased food intake in NaF-treated animals (Tsunoda et al., 2005; Nageshwar et al., 2017).

Exposure to fluoride causes a cognitive impairment along with failure of habituation in an open field task. Analysis of crossing, rearing, and sniffing responses showed that only the control group had the memory for habituation. The memory for habituation in the open field test is processed by the hippocampus. The reduced dopamine level leads to a decrease in the open field rearing and scratching behaviors in rats, which clearly shows an association between dopamine and complex stereotypic behaviors caused by fluoride intoxication. The effects of fluoride on brain neurochemistry are more pronounced in younger rats (Niu, Sun, Wang, Cheng, \& Wang, 2008). Shrinivas et al., (2008) reported that curcumin restored the locomotor activities altered by environmental toxicants.

Xu et al., (2005) also investigated the neuroprotective action of curcumin on altered behavioral activities in animal models. Another experimental study stated that curcumin improved memory and learning ability in rats (Mohit et al., 2009). Therefore, experimental animals during development exhibit more sensitivity to fluoride effect on learning, which could be due to intrinsic age-related differences in the molecular sites, at which fluoride interacts. The present results showed the reversal of the behavioral alteration induced by $\mathrm{NaF}$ in the developing brain of rats treated with curcumin that may be due to the modulation of the catecholamine neurotransmitter system.

Histopathological changes were seen in the hippocampus CA1 and CA2 regions of developing brain of rats. On days 14,21 , and 30 , the shrunken neurons with dark stained small nucleus showed a decrease in number and density in the developing brain of the rats exposed to NaF. All subregions of the hippocampus were affected by fluoride toxicity. Curcumin-administered groups showed healthy neurons and good cell arrangement and density compared with the NaF-treated group. The hippocampus showed drastic neurodegenerative changes in the NaF-treated group. Mullenix et al., (1995) reported the accumulation of fluoride in the hippocampus region. Varner et al. (1998) announced altered structures in the CA1 and CA4 areas of the hippocampus but not in CA2 and CA3 areas. Recently, Sudhakar et al., (2017) showed a histological alteration in the NaF-intoxicated brain of rats. Curcumin treatment caused a decrease in neurodegenerative effects (Chhavi, Pooja, Piyu, Neha, \& Maheep, 2014). Curcumin significantly reversed the environmental toxicants adverse effects on the diameter of glomerulus and proximal tubule in the kidney tissue of animals (Hamid, \& Najmeh, 2017). A significant increase in the viable neuronal cell was found in curcumin treatment possibly due to neuroprotection.

The earlier reports and our findings support that curcumin significantly ameliorated $\mathrm{NaF}$-induced changes in neurotransmitters and behavioral and histological alterations in the developing rat hippocampus. Curcumin restored the 
levels of neurotransmitters (epinephrine, norepinephrine, dopamine, and acetylcholine), and caused behavioral and histopathological alterations in the fluoride-treated pups. Among the doses of curcumin, both 10 and $20 \mathrm{mg} / \mathrm{kg}$ bw doses were effective; however, the dosage of $20 \mathrm{mg} / \mathrm{kg} \mathrm{bw}$ was more protective than $10 \mathrm{mg} / \mathrm{kg}$ bw. The curcumin possibly exhibits neuroprotection through the modulation of the neurotransmitter system and has antioxidant potential in the developmental toxicity of $\mathrm{NaF}$ in the hippocampus and behavior of the rats.

Our results demonstrated that developmental exposure of fluoride altered the neurotransmitters system and caused concomitant histological changes in the hippocampus, as well as behavior in rats. Curcumin, as a known anti-oxidant, has a progressive reversal effect against $\mathrm{NaF}$-induced acetylcholine, catecholamine, and histological alteration in the hippocampus in developing brain of the rats and altered behavior. Among the two doses of curcumin, a dosage of $20 \mathrm{mg}$ was more effective than $10 \mathrm{mg}$. Thus, supplementation of curcumin at the doses of 10 and $20 \mathrm{mg} / \mathrm{kg}$ bw has beneficial effects in ameliorating the neurotoxic effects of $\mathrm{NaF}$.

\section{Ethical Considerations}

\section{Compliance with ethical guidelines}

All experimental procedures were conducted in accordance with the guidelines of CPCSEA, India for care and use of laboratory animals.

\section{Funding}

This work was partially supported by a grant DBT project (Sanction Order No.BT/PR/14040/med/30/336/2010), New Delhi and UGC DSA-I (Lr.No.F.5-26/2015/DSA-I (SAP-II)/ Zoology/2015), program New Delhi.

\section{Authors' contributions}

All authors contributed equally in research design, conduction of experiment, resulting analysis and preparation of manuscript.

\section{Conflict of interest}

The authors declared no conflict of interest.

\section{Acknowledgments}

The authors acknowledge the partial financial support under the DBT project (Sanction Order No. BT/PR/14040/ med/30/336/2010), New Delhi, and UGC DSA-I (Lr.No.F.526/2015/DSA-I (SAP-II)/ Zoology/2015), New Delhi.

\section{Refrencess}

Arora, V. A., Kuhad, V., \& Tiwari, K. C. (2011). Curcumin ameliorates reserpine-induced pain-depression dyad: Behavioural, biochemical, neurochemical and molecular evidences. Psychoneuroendocrinology, 36(10), 1570-81. [DOI:10.1016/j.psyneuen.2011.04.012] [PMID]

Bhatnagar, M., Rao, P., Saxena, A., Bhatnagar, R., Meena, P., Barbar, S., \& Chouhan, A., et al. (2006). Biochemical changes in brain and other tissues of young adult female mice from fluoride in their drinking water. Fluoride, 39(4), 280-4.

Brown, L., Buckingham, S., \& Jones, A. (2009). Nicotinic Acetylcholine Receptor Signaling: Roles in Alzheimer's disease and Amyloid Neuroprotection. Pharmacological Review, 61(1), 39-61. [DOI:10.1124/pr.108.000562] [PMID] [PMCID]

Chandrashakar, R. N., \& Reddy, K.P. (2016). Protective effect of resveratrol against neuronal damage through oxidative stress in cerebral hemisphere of aluminum and fluoride treated rats. Interdisciplinary Toxicology, 9(2), 78-82. [DOI:10.1515/intox-2016-0009] [PMID] [PMCID]

Chhavi, S., Pooja, S., Piyu, S., Neha, J., \& Maheep, B. (2014). Curcumin attenuates neurotoxicity induced by fluoride: An in vivoevidence. Pharmacoghosy Magazine, 10(37), 61-5. [DOI:10.4103/09731296.126663] [PMID] [PMCID]

Chinoy, N., J., Sequeria, E., \& Narayana, M.V. (1991). Effects of vitamin $\mathrm{C}$ and calcium on the reversibility of fluoride induced alterations in spermatozoan of rabbit. Fluoride, 24(1), 29-39.

Chirumari, K., \& Reddy, K. P. (2007). Dose dependent effects of fluoride on neurochemical milieu in hippocampus and neocortex of rat brain. Fluoride, 40(2), 101-10.

Ekambaram, P., \& Vanaja, P. (2001). Environmental Toxicology and Pharmacology, 9, 141-6. [DOI:10.1016/S1382-6689(00)00063-6]

Geeraerts, F., Gijs, G., Finne, E., \& Crokaert, R. (1986). Kinetics of fluoride penetration in liver and brain. Fluoride, 19(3), 108-12.

Hamid, R. M., \& Najmeh, E. (2017). Effect of curcumin on kidney histopathological changes, lipid peroxidation and total antioxidant capacity of serum in sodium arsenite-treated mice. Ex perimental and Toxicologic Pathology, 69(2), 93-7. [DOI:10.1016/j. etp.2016.08.006] [PMID]

Hemlata, T., \& Mandava, V. R. (2010). Curcumin supplementation protects from genotoxic effects of arsenic and fluoride. Food and Chemical Toxicology, 48(5), 1234-8. [DOI:10.1016/j.fct.2010.02.015] [PMID]

Hestrin, S. (1949). The reaction of acetylcholine and other carboxylic acid derivatives with hydroxylamine, and its analytical application. Journal of Biological Chemistry, 180(1), 249-61.

Itmori, K., Tanaka, E. M., Kohno, Y., Ida, Y., Nakagawa, R., \& Thoshima, et al. (1982). Psychological stress enhances nor adrenaline turnover in specific brain regions in rats. Pharmacology Biochemistry and Behavior, 16(4), 637-40. [DOI:10.1016/0091-3057(82)90429-4]

Joyce, T., Yolanda, I. C., Eduardo, M. J., Ana Cristina A. R., Edilia, T., \& José P. C., et al. (2013). Renoprotective effect of the antioxidant curcumin: Recent findings. Redox Biology, 1(1), 448-56. [DOI:10.1016/j.redox.2013.09.003] [PMID] [PMCID]

Kari, H. P., Davidson, P. P., Kohl, H. H., \& Kochhar, M. M. (1978). Effects of ketamine on brain monoamine levels in rats. Research Communications in Chemical Pathology and Pharmacology, 20(3), 475-88. 
Li, J. X., \& Cao, S. R. (1994). Recent studies on endemic flourosis in china. Fluoride, 27, 125-8

Li, X, Z., \& Gao, R. (1995). Effect of fluoride exposure on intelligence in children. Fluoride, 28, 189-92.

Lu, Y., Sun Z. R., Wu, L. N., Wang, X., Lu, W., Liu, S. S. (2000). Study of cognitive functionimpairment caused by fluorosis. Fluoride, 33, 74-8.

Madhusudhan, N., Basha, P. M., Rai, P., Ahmed, F., \& Prasad, G. R (2010). Effect of maternal fluoride exposure on developing CNS of rats: Protective role of Aloe vera, Curcuma longa and Ocimum sanctum. Indian Journal Experimental Biology, 48(8), 830-6.

Mahendra, B., Kanwaljit, C., \& Shrinivas, K. K. (2008). Protective effect of Curcumin, the active principle of turmeric (Curcuma longa) in haloperidol-induced orofacial dyskinesia and associated behavioural, biochemical and neurochemical changes in rat brain. Pharmacology Biochemistry and Behavior, 88(4), 511-22. [DOI:10.1016/j. pbb.2007.10.009] [PMID]

Majid, M., Morteza, K., Ozra, M., Behnaz, S., Iraj, Y., \& Sulail, F., et al. (2015). Protective effects of various dosage of Curcumin against morphine induced apoptosis and oxidative stress in rat isolated hippocampus. Pharmacological Reports, 67(2), 230-5. [DOI:10.1016/j. pharep.2014.09.006] [PMID]

Marcela, P., Patricia, A. D., Estela, M. L., Lea, R. C., Claudio D. C., \& Roberto, A. (2011). Memory impairment induced by sodium fluoride is associated with changes in brain monoamine levels. Neurotoxicity Research, 19(1), 55-62. [DOI:10.1007/s12640-009-91395] [PMID]

Mohit, K, B., Mahendra, B., \& Shrinivas, K.K. (2009). Anti-depressant like effect of curcumin and its combination with piperine in unpredictable chronic stress-induced behavioral, biochemical and neurochemical changes. Pharmacology, Biochemistry and Behavior, 92(1), 39-43. [DOI:10.1016/j.pbb.2008.10.007] [PMID]

Mullenix, P.J., Denbesten, P.K., Schunior, A., \& Kernan, W. J. (1995) Neurotoxicity of sodium fluoride in rats. Neurotoxicology and Teratology, 17(2), 169-77. [DOI:10.1016/0892-0362(94)00070-T]

Nabavi, S. F., Eslami, Sh., Hajizadeh, M. A., \& Nabavi, S. M. (2011). Protective effects of curcumin against fluoride-induced oxidative stress in the rat brain. Neurophysiology, 43(4), 332-6. [DOI:10.1007/ s11062-011-9228-y]

Nabavi, S. F., Moghaddam, A. H., Eslami, S., \& Nabavi, S. M. (2012). Protective effects of curcumin against sodium fluoride-induced toxicity in rat kidneys. Biological Trace Element Research, 145(3), 369 74. [DOI:10.1007/s12011-011-9194-7] [PMID]

Nabavi, S. F., Moghaddan, A. H., Nabavi, S. M., \& Eslami, S. (2011) Protective effects of curcumin and quercetin on thyroid function in sodium fluoride intoxicated rats. Fluoride, 44(3), 147-52

Nabavi, S. F., Nabavi, S. M., Ebrahimzadeh, M. A., Eslami, S.H., Jafari, N., \& Moghad A. H. et al. (2011). The protective effect of curcumin against sodium fluoride-induced oxidative stress in rat heart. Archives of Biological Science Belgrade, 63(3), 563-9. [DOI:10.2298/ ABS1103563N]

Nageshwar, M., Kirankumar, N., Rajkiran R. B., Chandrashakar R. N., \& Reddy K. P. (2017). Quercetin treatment against NaFinduced oxidative stress related neuronal and learning changes in developing rats. Journal of King Saud University-Science, 29(2), 2219. [DOI:10.1016/j.jksus.2016.04.002]
Nageshwar, M., Sudhakar, K., Chandrashakar, N. R., \& Reddy K. P. (2017). Neuroprotective effects of curcumin on sodium fluoride induced behavioural and enzymatic changes in brain and muscles of rat. Journal of Environmental Biology, 38(4), 675-81. [DOI:10.22438/jeb/38/4/MS-169]

Netto, C. A., Dias, R. D., \& Izquiedo, I. (1986). Differenial effect of post training naloxone, beta-endorphin, leu-enkephalin and electroconvulsive shock administration upon memory of an openfield habituation and of a water-finding task. Psychoneuroendocrinology, 11(4), 437-46. [DOI:10.1016/0306-4530(86)90005-3]

Niu, R. Y., Sun, Z. L., Wang, J. M., Cheng, Z., \& Wang, J. D. (2008) Effects of fluoride and lead on locomotor behavior and expression of Nissl body in brain of adult rats. Fluoride, 41, 276-82.

Pilati, N., Matthew, B., sofoklis, P., Revers, D., \& Martine, H. (2008) A rapid method combining golgi and staining to study neuronal morphology and cytoarchitechture. Jouranl of Histochemistry and Cytochemistry, 56(6), 539-50. [DOI:10.1369/jhc.2008.950246] [PMID] [PMCID]

Rajkiran, R. B., \& Reddy, K. P. (2015). Vitamin A deficiency: An oxidative stress marker in sodium fluoride $(\mathrm{NaF})$ induced oxidative damage in developing rat brain. International Journal of Developmental Neuroscience, 47(Pt B), 298-03. [DOI:10.1016/j. ijdevneu.2015.08.010] [PMID]

Rajkiran, R. B., Kiran, K. N., Khalid, P. M., Manjula R. M., \& Pratap R. K. (2018). Carica Papaya leaf extract as a neuroprotective agent against behavioral and neurotransmitter changes in brain of the rat treated with sodium fluoride in pre- and post-natal periods. Pharmacognosy Magazine, 14(55), 123-31. [DOI:10.4103/pm.pm_378_17]

Seibenhener, M. L., \& Wooten, M. C. (2015). Use of the open field maze to measure locomotor and anxiety-like behavior in mice Journal of Visualized Experiments, (96):e52434. [DOI:10.3791/52434] [PMID] [PMCID]

Shamsher, S., Sumit, J., \& Puneet, K. (2015). Piperine enhances the protective effect of curcumin against 3-np induced neurotoxicity: Possible neurotransmitters modulation mechanism. Neurochemical Research, 40(8), 1758-66. [DOI:10.1007/s11064-015-1658-2] [PMID]

Shan, K. R., Qi, X. L., Long, Y. G., Nordberg, A., \& Guan, Z. Z. (2004). Decreased nicotinicreceptors in PC12 cells and rat brains influenced by fluoride toxicity-a mechanism relating to a damage at the level in post-transcription of the receptor genes. Toxicology, 200(2-3), 169-77. [DOI:10.1016/j.tox.2004.03.013] [PMID]

Shantakumari, D., Srinivasulu, S., \& Subramanian, S. (2004). Effect of fluoride intoxication on lipid peroxidation and antioxidant status in experimental rats. Toxicology, 204(2-3), 219-28. [DOI:10.1016/j. tox.2004.06.058] [PMID]

Shivarajashankara, Y. M., Shivashankara, A. R., Gopalakrishna, B P., \& Rao, S. H. (2001). Oxidative stress in children with endemic skeletal fluorosis. Fluoride, 34(2), 103-7.

Shrinivas, K. K., Mohit, K. B., \& Mahendra, B. (2008). Antidepressant activity of curcumin: Involvement of serotonin and dopamine system. Psychopharmacology, 201(3), 435-42. [DOI:10.1007/s00213008-1300-y] [PMID]

Sudhakar, K., Nageshwar, M., \& Reddy K. P. (2017). Seed extract of Abelmoschus moschatus medik reverses $\mathrm{NaF}$-induced behavioral changes through neurodegeneration and oxidative stress in brain of rat. Asian Journal of Pharmaceutical and Clinical Research, 10(10), 1-7. [DOI:10.22159/ajpcr.2017.v10i10.20215] 
Suresh, D., \& Srinivasan, K. (2007). Studies on the in vitro absorption of spice principles Curcumin, capsaicin and piperine in rat intestines. Food and Chemical Toxicology, 45(8), 1437-42. [DOI:10.1016/j. fct.2007.02.002] [PMID]

Tsunoda, A., Wakano, L., Horiuchi, Itai, \& Tsunoda. (2005). Changes in fluoride levels in the liver, kidney, brain and neurotransmitters of mice after subacute administration of fluoride. Fluoride, 38(4), 284-92.

Vani, L. M., \& Reddy, K. P. (2000). Effect of fluoride accumulation on some enzymes of brain and gastrocnemius muscles of mice. Fluoride, 33(1), 17-26.

Varner, J. A., Jensen, K. F., Horvath, W., \& Issacon, R. L. (1998). Chronic administration of aluminium fluoride or sodium fluoride to rats in drinking water: Alterations in neuronal and cerebrovascular integrity. Brain Research, 784(1-2), 284-98. [DOI:10.1016/ S0006-8993(97)01336-X]

Wang, J., Ge, Y., Ning, H., \& Wang, S. (2004). Effects of high fluoride and low iodine on oxidative stress and antioxidant defense of the brain in offspring rats. Fluoride, 37(4), 264-70.

Wu, C., Gu, X., Ge, Y. M., Zhang, J., Wang, J. D. (2006). Effects of high fluoride and arsenic on brain biochemical indexes and learningmemory in rats. Fluoride, 39(4), 274-9.

Xiaolie, H., Yanjing, Z., Mei, W., Guoxin, J., Rongrong, Z. \& Shilong, W., et al. (2016). Antidepressant effects of curcumin and HU-211 coencapsulated solid lipid nanoparticles against corticosterone induced cellular and animal models of major depression. International Journal of Nanomedicine, 11, 4975-90. [DOI:10.2147/IJN. S109088] [PMID] [PMCID]

Xu, Y., Ku, B. S., Yao, H. Y., Lin, Y. H., Ma, X., Zhang, Y. H., Li, X.J. (2005). The effects of curcumin on depressive-like behaviors in mice. European Journal of Pharmacology, 518(1), 40-6. [DOI:10.1016/j. ejphar.2005.06.002] [PMID]

Ying, X., Bao-Shan, K., Hai-Yan, Y., Yan-Hua, L., Xing, M., \& Yong$\mathrm{He}$, Z. et al. (2005). Antidepressant effects of curcumin in the forced swim test and olfactory bulbectomy models of depression in rats. Pharmacology Biochemistry and Behavior, 82(1), 200-6. [DOI:10.1016/j.pbb.2005.08.009] [PMID]

Yugandhar, R. P., Reddy, K. P., \& Praveen, K. K. (2011). Neurodegenerative changes in different regions of brain, spinal cord and sciatic nerve of rats treated with sodium fluoride. Journal of Medical E Allied Sciences, 1(1), 30-5. 
This Page Intentionally Left Blank 